\title{
Evaluations of the solution to the homogeneous plane problem of the theory of elasticity in the neighborhood of an irregular boundary point
}

\author{
Ludmila Frishter ${ }^{1, *}$ \\ ${ }^{1}$ Moscow state university of civil engineering, Yaroslavskoye shosse, 26, Moscow, Russia, 129337
}

\begin{abstract}
The most complex stress-strain state (SSS) occurs in the domain of stress concentration due to the shape of the boundary ("the geometric factor") and the finite discontinuities of the specified forced deformations, the mechanical properties, emerging at the irregular point of the boundary of the domain. In this paper, there is a review of the methods for analyzing the particular qualities of the solution to the problem of the theory of elasticity, due to the shape of the boundary or "the geometric factor". The features of the stress-strain state of constructions and structures, possessing "constructive heterogeneity" under the action of discontinuous forced deformations, are stress concentrators, determined on the polymer models of the photoelastic method. For interpreting and decoding of experimentally obtained local SSS in stress concentration domains of structures there are given estimations of the solution to homogeneous plane problem of the elasticity theory in neighborhood of an irregular boundary point.
\end{abstract}

\section{Introduction}

The stress-strain state (SSS) of complex structures is characterized by a significant concentration of stresses in the domains of conjugation of elements with different options for the constructive design of the boundary: singular lines, incoming angle, etc.

The research of the stress state of complex structures in the domains of conjugation of elements made from materials with different mechanical properties under the action of forced deformations, that are discontinuous along the contact line (surface) of the elements, is an actual problem in the practice of engineering design. The research of the stress-strain state of complex structures in zones of constructive heterogeneity is focused on the consideration of the problem of elasticity theory in the neighborhood of the irregular point of the domain boundary, which includes the discontinuity (jump) of forced deformations.

\footnotetext{
*Corresponding author: $\underline{\text { frishter@mail.ru }}$
} 


\section{Methods for analyzing the solution to elastic problems in the neighborhood of an irregular boundary}

2.1. Questions of the behavior of solutions to the Laplace, Poisson, and elliptic equations for domains with non-smooth boundaries are considered by V.A. Kondratyev, V.V. Fufaev, M.L. Williams, S.Ja. Ufljand, A.I. Kalandii, G.P. Cherepanov, D.B. Bodzhy, O.K. Aksentjan, A.Ja. Aleksandrov, K.S. Chobanjan, I.T. Denisjuk, V.D. Kuliev, etc.

The fundamental work of V.A. Kondratiev [1] proves that the solution to the general elliptic boundary value problem in the neighborhood of irregular points of the domain boundary is represented in the form of an asymptotic expansion and an infinitely differentiable function. The terms of this expansion contain solutions to homogeneous boundary value problems for model domains: a wedge or a cone. They depend on the local characteristics - the magnitude of the solid or flat angle and the type of boundary conditions, the mechanical characteristics for piecewise homogeneous bodies. The magnitudes of the coefficients to the solution expansion in the neighborhood of a singular point are unknown and depend on the problem. The methods for determining these coefficients of decomposition are complex and difficult to be realized in the practical determination of stresses of complex structures having a complex shape of the boundary.

In the scientific papers of I.T. Denisyuk is given the asymptotics of the elastic solution to a plane complex domain with angular points on the separation lines [2]. The work of V.D. Kuliev [3] shows the advantage of a fundamental research of the singular solution to the boundary value problem in the possibility of applying its results to the investigation of problems on residual stresses and crack propagation across the connection boundary of dissimilar materials. It is shown that the order of the stress singularity depends on the approach degree of the crack tip to the connection domain of materials with different Young's modulus.

Mostly referred works of M.L. Williams show that stresses, deformations, the Erie stress function near the vertex of the sector with rectilinear sides have got a polynomial form [4]. The works of A.I. Kalandii, K.S. Chobanjan, L.A. Bagirov, O.K. Aksentjan, G.P. Cherepanov, V.P. Netrebko and many others represent the solution to a homogeneous boundary value problem in a neighborhood of an irregular boundary point in a polynomial form.

2.2. The scientific work [5] introduces a local curvilinear coordinate system to research the SSS in the neighborhood of an irregular point on the singular boundary line of an elastic body in which the Lame equations are written in. When approaching to an irregular point of the boundary from the inside the solution to the elastic problem comes down to solving two homogeneous plane problems: plane deformation and antiplane deformation or a transverse shift. It is possible to show that the representation of the solution to an elastic problem in the neighborhood of an irregular point on a singular boundary line in the form of two homogeneous plane problems is valid in the case when: a) given forced deformations and body forces are continuous along the domain of the elastic body; b) given forced deformations and body forces are piecewise-continuous functions, and moreover, the jump in the values of the forced deformations and body forces along the inner surface of the contact of the domains goes to the specific boundary line of the body; c) the contact surface of domains $V_{1}$ and $V_{2}$ of the elastic composite body $V$, having different mechanical characteristics $E_{i}, v_{i}, i=1,2$, goes to the specific boundary line of the body. In case of c), the solution to the elastic problem in the neighborhood of an irregular point of the boundary comes down to solving plane problems for a composite body. 
2.3. In $[6,7,8]$, similarity theory is used to research the solution to an elastic problem in displacements in the neighborhood of an irregular point on a singular boundary line of the domain.

We consider a small neighborhood of an irregular point on a singular line - the line of discontinuity, for example, boundary conditions or the first derivatives of the surface function of the domain. In a small neighborhood of the irregular point of the surface boundary, there is applied the following similarity group: $x_{1}=t x ; y_{1}=t y ; z_{1}=z ; t>0$, when $t$ is the group parameter. Writing the Lame equation in a small neighborhood of a point on a singular line and passing to the limit for $t \rightarrow+\infty$, the solution to the elastic problem comes to solving two homogeneous plane problems, as in case 2.2: a plane deformation and an antiplane deformation.

In [6], the concept of a canonical singular problem characterizing the singularity of the SSS in the neighborhood of an irregular boundary is defined, for which the following two theorems are valid:

1) Any canonical singular problem corresponds to a transcendental equation, to each root of which there is corresponded a homogeneous solution, the number of arbitrary real constants in this solution is equal to the multiplicity of the root.

2) In an infinitesimal neighborhood of a singular point the solution to the correct boundary value problem of the theory of elasticity behaves as an asymptotically largest in absolute value eigenfunction of the corresponding canonical singular problem.

\section{Statement of the problem and estimations of the solution}

We consider the solution to the problem of elasticity theory for a homogeneous or piecewise homogeneous body in the neighborhood of an irregular point of the boundary of a plane domain into which a finite discontinuity (jump) of the forced deformations is included.

According to the theoretical analysis [8], the solution to the problem of elasticity theory in the neighborhood of an irregular point of the boundary of a plane domain can be written in the following form:

or

$$
\begin{gathered}
\sigma_{i j}=\sigma_{i j}^{E}+\sigma_{i j}^{S}, \quad \varepsilon_{i j}=\varepsilon_{i j}^{E}+\varepsilon_{i j}^{S}, \quad U_{i}=U_{i}^{E}+U_{i}^{S}, \\
\eta=\eta^{E}+\eta^{S},
\end{gathered}
$$

when $\eta^{E}=\left(\sigma_{i j}^{E}, \varepsilon_{i j}^{E}, U_{i j}^{E}\right)$ - "eigen" solution to a homogeneous boundary value problem in a neighborhood of an irregular point of the domain boundary characterizes the singularity for the solution;

$\eta^{S}=\left(\sigma_{i j}^{S}, \varepsilon_{i j}^{S}, U_{i j}^{S}\right)$ - the stress-strain state, determined by the action of given loads, depends on the geometric parameter - the "approximation degree" to the singular point. The representation (1) is also valid in the spatial case for the points on the singular boundary line of the domain.

We consider a small neighborhood of the irregular point $O$ on the singular boundary line of $V$ - body, as a self-balanced part of body $V$ in the following form:

$x^{2}+y^{2}<\varepsilon_{1}^{2} ; z^{2}<\varepsilon_{2}^{2} ; \varepsilon_{1}, \varepsilon_{2}$ - are small positive numbers.

The conditions on boundary $L$ of the domain are homogeneous.

The original elastic problem in the neighborhood of an irregular boundary point admits a similarity group:

$$
x_{1}=t x ; \quad y_{1}=t y ; \quad z_{1}=z
$$




$$
\sigma_{i j}=t \overline{\sigma_{i j}} ; \quad \varepsilon_{i j}=t \overline{\varepsilon_{i j}} ; \quad U_{i}=\overline{U_{i}},
$$

when $i, j=x, y, z ; i 1, j 1=x_{1}, y_{1}, z_{1} ; t$ - is a group parameter, $t>0$.

In an infinitesimal neighborhood of a singular point of the boundary, the solution to the correct boundary value problem of elasticity theory behaves as the asymptotically largest one in absolute value eigenfunction of the corresponding canonical singular problem. The singularity of stresses and strains has order $r^{\lambda_{0}-1}$, when $\lambda_{0}=\min \operatorname{Re} \lambda$ - the minimal value of the real part of the complex root of the characteristic equation of a homogeneous plane boundary value problem of elasticity theory is determined numerically [9].

We choose a neighborhood $c<r<(1+\alpha) c, \quad \alpha \in(0,1), \quad \theta \in\left(\theta_{0}, \theta_{1}\right)$ in which the nonsingular plane homogeneous problem with homogeneous boundary conditions is valid. The parameter $t=\frac{1}{c}$ is great enough and parameter $c$ is small enough so as not to take into account the stresses of the common field $\sigma_{i j}^{S}$ determined by the given forced deformations.

The stress function of a homogeneous plane elastic problem in a polar coordinate system is written in the following form:

$$
\varphi=r^{\lambda_{0}+1} f(\theta)
$$

when $\lambda_{0}=\min \operatorname{Re} \lambda-$ the minimum value of the real part of the complex root $\lambda$ of the characteristic equation of a homogeneous boundary (singular) problem in the domain $r<\varepsilon_{1}<c ; \varepsilon_{1}>0, f(\theta)$ - function of the variable $\theta, \theta \in\left(\theta_{0}, \theta_{1}\right)$.

We introduce a new variable $r_{1}=r-c, \quad r \in[c,(1+\alpha) c], \quad r_{1} \in(0, \alpha c)$. The stress function (2) is taken in the following form:

$$
\varphi=\left(r_{1}+c\right)^{\lambda_{0}+1} f(\theta)=c^{\lambda_{0}+1}\left(1+\frac{r_{1}}{c}\right)^{\lambda_{0}+1} f(\theta),
$$

when $0<\frac{r_{1}}{c}<\alpha<1$. We apply the binomial expansion in the following form:

$$
\begin{aligned}
& \left(1+\frac{r_{1}}{c}\right)^{\lambda_{0}+1}=1+\left(\lambda_{0}+1\right)\left(\frac{r_{1}}{c}\right)+\frac{\left(\lambda_{0}+1\right) \lambda_{0}}{2 !}\left(\frac{r_{1}}{c}\right)^{2}+ \\
& +\frac{\left(\lambda_{0}+1\right) \lambda_{0}\left(\lambda_{0}-1\right)}{3 !}\left(\frac{r_{1}}{c}\right)^{3}+\ldots+\frac{\left(\lambda_{0}+1\right) \lambda_{0}\left(\lambda_{0}-1\right) \cdots\left(\lambda_{0}-n+2\right)}{n !}\left(\frac{r_{1}}{c}\right)^{n} \ldots
\end{aligned}
$$

The stress function (3) takes into account the expansion (4) and is rewritten in the following way:

$$
\varphi=c^{\lambda_{0}+1}\left[1+\left(\lambda_{0}+1\right)\left(\frac{r_{1}}{c}\right)+\sum_{n=2}^{\infty} \frac{\left(\lambda_{0}+1\right) \lambda_{0}\left(\lambda_{0}-1\right) \cdots\left(\lambda_{0}-n+2\right)}{n !}\left(\frac{r_{1}}{c}\right)^{n}\right] f(\theta) .
$$

According to the stress function (5), we write the stresses in the neighborhood of the irregular point of the boundary as follows: 


$$
\begin{aligned}
& \sigma_{r}=\frac{1}{r} \frac{\partial \varphi}{\partial r}+\frac{1}{r^{2}} \frac{\partial^{2} \varphi}{\partial \theta^{2}}=\frac{c^{\lambda_{0}+1}}{r} f(\theta)\left[\frac{\lambda_{0}+1}{c}+\frac{\left(\lambda_{0}+1\right) \lambda_{0}}{c^{2}}(r-c)+\right. \\
& \left.+\sum_{n=3}^{\infty} \frac{\left(\lambda_{0}+1\right) \lambda_{0}\left(\lambda_{0}-1\right) \cdots\left(\lambda_{0}-n+2\right)}{(n-1) ! c^{n}}(r-c)^{n-1}\right]+\frac{c^{\lambda_{0}+1}}{r^{2}} f^{\prime \prime}(\theta)\left[1+\frac{\left(\lambda_{0}+1\right)}{c}(r-c)+\right. \\
& \left.+\sum_{n=2}^{\infty} \frac{\left(\lambda_{0}+1\right) \lambda_{0}\left(\lambda_{0}-1\right) \cdots\left(\lambda_{0}-n+2\right)}{n ! c^{n}}(r-c)^{n}\right], \\
& \sigma_{\theta}=\frac{\partial^{2} \varphi}{\partial \theta^{2}}=c^{\lambda_{0}+1} f(\theta)\left[\sum_{n=2}^{\infty} \frac{\left(\lambda_{0}+1\right) \lambda_{0}\left(\lambda_{0}-1\right) \cdots\left(\lambda_{0}-n+2\right)}{(n-2) ! c^{n}}(r-c)^{n-2}\right], \\
& \tau_{r \theta}=-\frac{\partial}{\partial r}\left(\frac{1}{r} \frac{\partial \varphi}{\partial \theta}\right)=-c^{\lambda_{0}+1} f^{\prime}(\theta)\left[\frac{\lambda_{0}}{r}+\right. \\
& \left.+\sum_{n=2}^{\infty} \frac{\left(\lambda_{0}+1\right) \lambda_{0}\left(\lambda_{0}-1\right) \cdots\left(\lambda_{0}-n+2\right)}{n ! c^{n}}\left(n-1+\frac{c}{r}\right) \frac{(r-c)^{n-1}}{r}\right]
\end{aligned}
$$

We estimate the remainder term of the series (5) in the following form:

$$
\left|R_{n}\left(\frac{r_{1}}{c}\right)\right|<\left|\frac{\left(\lambda_{0}+1\right) \lambda_{0}\left(\lambda_{0}-1\right) \cdots\left(\lambda_{0}+1-n\right)}{(n+1) !}\left(\frac{r_{1}}{c}\right)^{n+1}\right|<\delta,
$$

when $\frac{r_{1}}{c}=\frac{r-c}{c}<\alpha \ll 1, \lambda_{0} \in(0,1) ; \quad \alpha \in(0,1)$,

$$
\text { or } \quad\left|R_{n}\left(\frac{r_{1}}{c}\right)\right|<\left|\frac{\left(\lambda_{0}+1\right) \lambda_{0}\left(\lambda_{0}-1\right) \cdots\left(\lambda_{0}+1-n\right)}{(n+1) !}(\alpha)^{n+1}\right|<\delta \text {. }
$$

With an increase in the angle of the solution between the tangents drawn to the boundary of the domain at the singular point: $\lambda_{0} \rightarrow 0,5$. To assess the remainder $R_{n}\left(\frac{r}{c}\right)$ we consider, as an example, $\lambda_{0}=0,5$ :

For $n=0 \quad\left|R_{n}\right|<\frac{3}{2}(\alpha)<\frac{3}{2}$.

For $n=1 \quad\left|R_{n}\right|<\frac{3}{8}(\alpha)^{2}<\frac{3}{8}=0,375$.

For $n=2 \quad\left|R_{n}\right|<\frac{9}{48}(\alpha)^{3}<0,09375<0,1$.

Therefore, with the necessary accuracy for the stress function, it suffices to take the first two (three) terms of the expansion series for $n=0,1$ (2), i.e.

or

$$
\varphi\left(r_{1}, \theta\right) \cong c^{\lambda_{0}+1} f(\theta)\left[1+\left(\lambda_{0}+1\right)\left(\frac{r_{1}}{c}\right)\right], \quad n=0,1,
$$

$$
\varphi(r, c) \cong c^{\lambda_{0}+1} f(\theta)\left[-\lambda_{0}+\left(\lambda_{0}+1\right) \frac{r}{c}\right]
$$

Taking into account the stress function (7) in the neighborhood $c<r<(1+\alpha) c, \quad c \in(0,1), \quad \alpha \in(0,1)$, the stresses (6) are written as following: 


$$
\sigma_{r} \cong \frac{c^{\lambda_{0}}}{r}\left(\lambda_{0}+1\right) c_{1}\left[f(\theta)+f^{\prime \prime}(\theta)\right], \quad \bar{\sigma}_{\theta}^{c} \cong 0 ; \quad \bar{\tau}_{r \theta}^{c} \cong 0 .
$$

The terms causing a significant increase in stresses and deformations (an increase in the deformation energy in the neighborhood: $r \in(0, \alpha c) ; \quad \alpha c \ll 1 ; \frac{1}{r^{2}} \gg \frac{1}{r}$; ) are not considered.

For example, for the stress function $f(\theta)=\left[c_{0} \cos \left(\lambda_{0}-1\right) \theta-c_{1} \cos \left(\lambda_{0}+1\right) \theta\right]$ at the first approximation the stresses will be written down in the following way:

$$
\sigma_{r} \cong \frac{c^{\lambda_{0}} c_{1}}{r}\left(\lambda_{0}+1\right) \lambda_{0}\left[c_{2}\left(2-\lambda_{0}\right) \cos \left(\lambda_{0}-1\right) \theta+\left(2+\lambda_{0}\right) \cos \left(\lambda_{0}+1\right) \theta\right],
$$

when $c_{1}, c_{2} \in \mathbb{R}$ - the arbitrary constants,

$$
\text { or } \sigma_{r} \cong \frac{\left(\lambda_{0}+1\right) \lambda_{0}}{r} c\left[c_{2}\left(2-\lambda_{0}\right) \cos \left(\lambda_{0}-1\right) \theta+\left(2+\lambda_{0}\right) \cos \left(\lambda_{0}+1\right) \theta\right], \quad \sigma_{\theta} \cong 0 ; \quad \tau_{r \theta} \cong 0 \text {. }
$$

Choosing the required number of terms in the expansion series of the stress function (5), it is possible to obtain stresses and strains in the domain of the nonsingular solution to the problem with an accuracy corresponding to the accuracy of the data measurement by the photoelasticity method $[10,11]$. The neighborhood of the irregular point of the boundary of a plane domain, where the estimations of the nonsingular solution to the homogeneous problem (5), (7), (6) are valid, are chosen from the experimental data.

In accordance with the accuracy of the photoelasticity method [10], it is sufficient to take the first two (three) terms of the series (5) for the expansion of the stress function for $\mathrm{n}$ $=0,1(2)$.

\section{Conclusions}

In the solution domain to a nonsingular homogeneous plane elastic problem, there is a possible estimation to the solution, using which we can extrapolate the solution to sections close to an irregular boundary point, taking into account the experimental data and practical accuracy of the measurement by the photoelasticity method.

\section{References}

1. V.A. Kondratyev. Proceedings of the Moscow Mathematical Society. Vol.16. pp. 209-292 (1967)

2. I. T. Denisjuk. Proceedings of universities, Mathematics, 6, pp.70-74 (2000)

3. V.D. Kuliev. M.:Science, Singular boundary value problems. p.719 (2005)

4. M. L. Williams. J. Appl. Mech., v. 19, 4, p. 526 (1952)

5. O.K. Aksentian. Applied Mathematics and Mechanics., 1967, Vol.31, Issue 1, pp.178-186 (1967)

6. G.P. Cherepanov. M.:Science, Mechanics of brittle fracture. p.640 (1974)

7. G.S.Vardanjan, L.Ju.Frishter. International journal for computational civil and structural engineering. Volume 3, Issue 2, pp.75-81 (2007)

8. L.Ju.Frishter. Proceedings of MGSU, 1, pp.169-174 (2008)

9. G.S.Vardanjan, M.L. Mozgaleva, V.N. Savostyanov, L.Ju.Frishter. Proceedings of universities.Civil Engineering. 10, pp.28-31 (2003) 
10. G.L. Hesina and others. M.: Stroyizdat, The photoelasticity method. In three volumes. Vol.3, p.311 (1975)

11. G.S. Vardanjan, V.N. Savostyanov, L.Ju. Frishter. The development of methods of experimental mechanics. Under. ed. O.N. Makhutova and others.- M.: IMASH RAS, pp.6068 (2003) 